\title{
Melatonin signalling and type 2 diabetes risk: too little, too much or just right?
}

\author{
Hindrik Mulder ${ }^{1}$
}

Received: 8 November 2016 / Accepted: 6 February 2017 /Published online: 16 March 2017

(C) Springer-Verlag Berlin Heidelberg 2017

\begin{abstract}
Of the associations of genetic variants with type 2 diabetes, the one of an SNP in an intron of the gene encoding the melatonin receptor 1B $(M T N R 1 B)$ has been remarkably robust. Work from our group and others has provided support for a model where carriers of this risk $G$ allele exhibit increased MTNR1B expression in islets of Langerhans. Most published studies to date favour that melatonin's action on the beta cell is inhibition of insulin secretion. Hence, our model proposes that this inhibitory effect of melatonin is exaggerated in carriers of the MTNR1B risk $\mathrm{G}$ allele. This would explain why this genetic association causes reduced insulin secretion and greater risk of future type 2 diabetes, as has been observed in numerous studies. Concurrently, another body of work has shown that rare MTNR1B alleles, which could perturb receptor function, also associate with type 2 diabetes. In this commentary, it is suggested that such apparently conflicting observations can be reconciled by the fact that non-coding (intronic; frequent) and coding (exonic; rare) alleles of MTNR1B give rise to different phenotypes. Thus, altered gene transcription may explain why SNPs, which do not alter coding sequences, exhibit cell-specific effects. In contrast, SNPs that change protein sequences are more likely to exert generalised effects since an altered protein will appear in all cells expressing the gene.
\end{abstract}

Hindrik Mulder

hindrik.mulder@med.lu.se

1 Unit of Molecular Metabolism, Lund University Diabetes Centre, Jan Waldenströms gata 35, SE-205 02 Malmö, Sweden
Keywords cAMP · Genome-wide association study $\left(\right.$ GWAS) · G-protein-coupled · Inhibitory G-protein $\left(\mathrm{G}_{\mathrm{i}}\right)$. Insulin secretion · Islet · Melatonin · Quantitative trait locus · Receptors $\cdot$ Single nucleotide polymorphism (SNP)
Abbreviations
eQTL Expression quantitative trait locus
FOXA2 Forkhead box A2
GWAS Genome-wide association studies
MTNR1A Melatonin receptor 1A
MTNR1B Melatonin receptor 1B
NEUROD1 Neurogenic differentiation 1

\section{Introduction}

Balance and dynamics are inherent and essential to endocrine systems. Endocrine regulation is rarely an on/off event; disease evolves when the balance is off-set. Levels of hormones may increase or decrease, as may signalling from hormones. Many pathways therefore exist whereby endocrine diseases develop. The archetype is provided by the thyroid: hypersecretion of thyroxine leads to thyrotoxicosis while abolished secretion causes hypothyroidism with myxoedema or cretinism.

Not every hormone has been definitively linked to common diseases, for example, melatonin. As a medical student, I was taught that melatonin was a vestigial hormone lacking a clear physiological role. It is an interesting twist of fate that I am now, as a scientist, engaged in elucidating the pathophysiological role of melatonin. Since my training in medical school, the mechanisms of circadian rhythm have 
largely been resolved and melatonin's role as the body's reporter of darkness has been established. In this issue of Diabetologia, Forrestel et al have reviewed chronomedicine in the context of type 2 diabetes [1]. Here, I will address a recent controversy about the role of melatonin receptors in the development of type 2 diabetes [2, 3].

\section{Melatonin and control of insulin secretion}

Until the late 2000s, the role of melatonin in insulin release was the concern of just a few groups. Peschke and colleagues have been leaders in this small field; extensive reviews of their work are available [4]. The two functional melatonin receptors (MT1 and MT2 in mice; melatonin receptor 1A [MTNR1A] and melatonin receptor $1 \mathrm{~B}$ [MTNR1B] in humans) are expressed in rodent islets, clonal beta cells [5] and in human islets $[6,7]$. As in other cell systems, both melatonin receptors couple to inhibitory G-proteins $\left(\mathrm{G}_{\mathrm{i}}\right)$, resulting in a reduction in cAMP levels upon activation. Since cAMP is a confirmed potentiator of insulin secretion, the effect of melatonin on insulin secretion has largely been reported as inhibitory $[4,8]$. This notwithstanding, stimulatory effects of melatonin on insulin secretion under certain conditions, particularly following prolonged stimulation [9] and in human islets [6], have been reported. A possible explanation is that the melatonin receptors also couple to other G-proteins, such as $\mathrm{G}_{\mathrm{q} / 11}$, mediating a stimulatory effect on insulin secretion via the phospholipase $\mathrm{C}$ system raising inositol trisphosphate $\left(\mathrm{IP}_{3}\right)$ [9], or that melatonin stimulates glucagon secretion [6].

\section{MTNR1B and the genetics of type 2 diabetes}

In the early days of genome-wide association studies (GWAS) of type 2 diabetes, we and others identified a common $M T N R 1 B$ allele that associates with fasting hyperglycaemia, decreased insulin secretion and increased risk of future type 2 diabetes [10-12]. This association has been remarkably robust, being replicated in several cohorts. In addition, Froguel's group identified a number of rare MTNR1B alleles, which have a stronger effect on type 2 diabetes risk [13]. These rare alleles, predicted to mediate loss-of-function, are discussed by Amélie Bonnefond [3].

\section{A model for the pathogenetic effect of the common $M T N R 1 B$ risk allele in type 2 diabetes}

We have attempted to resolve the pathogenetic mechanisms conferred by the common MTNR1B risk variant containing a $\mathrm{G}$ allele, i.e. the rs10830963 SNP [2]. Confirming previous preliminary data [10], RNA sequencing showed that rs10830963 is an expression quantitative trait locus (eQTL) in human islets (Fig. 1): the more $\mathrm{G}$ alleles one carries, the higher the mRNA expression of MTNR1B. Accordingly, overexpression of MTNR1B in clonal beta cells results in a stronger inhibition of insulin secretion exerted by melatonin. This was likely the result of a reduced rise in cAMP. Concurrent with this, we demonstrated that melatonin inhibits cAMP-formation in single clonal beta cells and that the cAMP response is retarded when MTNR1B is overexpressed [2]. Conversely, mice lacking the melatonin 2 receptor (MT2; i.e. the rodent form of MTNR1B) exhibit a greater fold-response of insulin secretion from islets. These mice also possess a greater number of islets, which contain a higher basal level of cAMP, and respond with a more rapid cAMP rise upon glucose stimulation. Consequently, islets from mice with Mt2 (also known as Mtnrlb) knockout release insulin more vigorously in vivo and in vitro in response to glucose. Daily administration of $4 \mathrm{mg}$ melatonin for 3 months to human homozygous carriers of the MTNR1B $\mathrm{G}$ allele results in a further decrease in glucose tolerance and attenuation of the insulin response [2].

Recent work has shed light on how the eQTL in MTNR1B may arise [14]: forkhead box A2 (FOXA2) binding sites, which are enriched across type 2 diabetes GWAS, can also be found at the MTNR1B locus. This is thought to enable binding of FOXA2 and/or other transcription factors to an enhancer region. Indeed, the transcription factor neurogenic differentiation 1 (NEUROD1) binds to rs 10830963 in the human fetal beta cell line EndoC- $\beta \mathrm{H} 1$, where the MTNR1B risk $\mathrm{G}$ allele creates a NEUROD1 consensus binding site. Accordingly, the region surrounding rs10830963, comprising the risk $\mathrm{G}$ allele, shows increased enhancer activity in EndoC- $\beta \mathrm{H} 1$ cells.

\section{Can gain- and loss-of-function of the MTNR1B locus in type 2 diabetes be reconciled?}

The fact that both gain- and loss-of function with respect to melatonin signalling have been suggested to contribute to the pathogenesis of type 2 diabetes has caused some controversy $[2,3,10,13]$. Without challenging the actual data, two possible scenarios could be considered (Fig. 2). First, alleles that change protein coding, albeit rare, will affect all cells expressing the gene, producing a potentially complex phenotype, which will be a composite of altered function in all cell systems expressing the gene. In contrast, a non-coding allele is more likely to affect gene regulation, i.e. binding of transcription factors and enhancers/repressors, as well as altering methylation, splicing and chromatin structure. Such mechanisms are subject to cell- and tissue-specific control and could explain the impact of rs10830963, comprising the G 


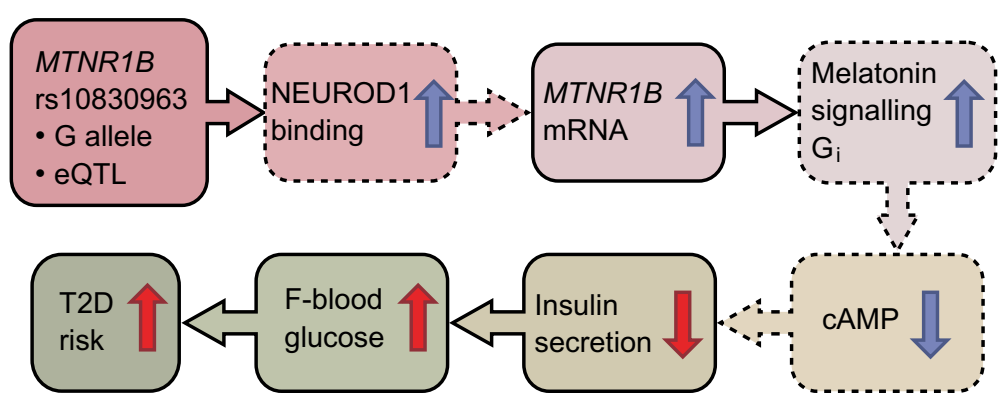

Fig. 1 A pathogenetic model for gain-of-function of melatonin signalling in islets conferred by a non-coding MTNRIB allele. In carriers of the rs $10830963 \mathrm{G}$ allele in the gene encoding the MTNR1B, an eQTL is created. This potentially allows increased binding of NEUROD1 to an enhancer region in which the rs10830963 SNP sits, hereby increasing expression of MTNR1B mRNA. This presumably leads to increased melatonin signalling via inhibitory G-proteins $\left(G_{i}\right)$. Consequently, formation of cAMP is hindered, resulting in less potentiation of insulin secretion. Fasting blood glucose (F-blood glucose) levels rise and the risk of future type 2 diabetes (T2D) increases. Dashed outline of boxes indicates that data are derived from experiments relevant to the model, e.g. from clonal beta cells or mouse islets, but not from human islets allele, on MTNR1B expression in beta cells and the ensuing inhibition of insulin release [15]. Second, returning to my introductory remarks, both abrogation and enhancement of melatonin action could be adverse for cellular function, particularly in a chronic situation, thus contributing to type 2 diabetes pathogenesis.

\section{Future directions for the study of melatonin signalling and metabolic control}

Several outstanding questions remain regarding melatonin signalling and metabolic control. Although we have shown that MTNRIB is more highly and abundantly expressed in islets than MTNR1A, with a preponderance for beta cells [16] (as largely supported by recent RNA sequencing efforts [7]) a redundancy of MTNR1A actions remains. Thus, the precise role of MTNR1A and MTNR1B must be teased out. Cell-specific targeting of MTNR1B and MTNR1A would be helpful in this respect. Conversely, overexpression of MTNR1B in beta cells should replicate the situation in G allele risk carriers. Moreover, the effects of melatonin in human islets have not been thoroughly examined: does melatonin inhibit insulin secretion in human islets?

Another important question is whether melatonin receptors are expressed in insulin target tissues. If so, is their expression changed in risk allele carriers and does this have an effect on metabolism in, for example, skeletal muscle, fat tissue and liver? Using genome editing technology to prove that the rs $10830963 \mathrm{G}$ allele is causal and confers the eQTL will be important. This can be accomplished in induced pluripotent stem cells from individuals carrying either the $\mathrm{G}$ allele or the $\mathrm{C}$ allele. Finally, controlled clinical studies of the effects of melatonin on metabolic regulation in humans are urgently needed.

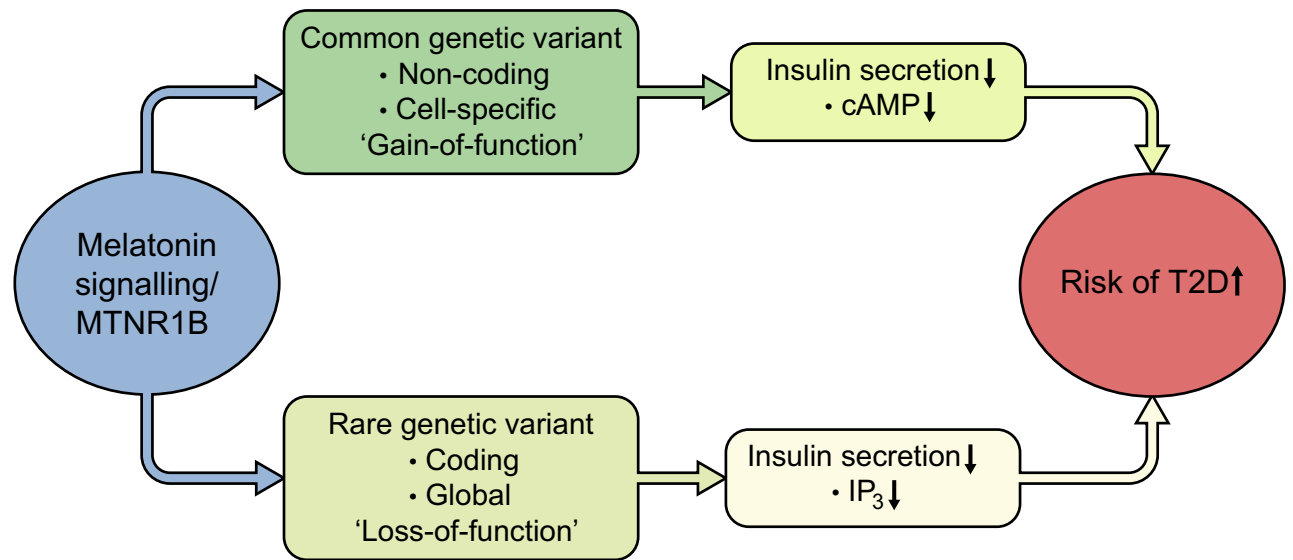

Fig. 2 Reconciling gain- and loss-of-function with respect to melatonin signalling. In the pancreatic beta cell, decreased insulin secretion could be mediated both by increased (common allele) and decreased (rare allele) signalling of melatonin. MTNR1B has been linked to both reduced formation of cAMP, via inhibitory G-proteins $\left(\mathrm{G}_{\mathrm{i}}\right)$, and to increased formation of inositol trisphosphate $\left(\mathrm{IP}_{3}\right)$, via $\mathrm{G}_{\mathrm{q} / 11}$; the former will reduce insulin secretion while the latter would increase secretion. In addition, a rare coding MTNRIB allele would likely affect all cells in which it is expressed, thereby enabling multi-organ mechanisms potentially leading to glucose intolerance and ultimately type 2 diabetes (T2D). In contrast, a non-coding allele of MTNRIB can be envisioned to exhibit a more cell-specific phenotype, since it will only become penetrant in cells that are equipped with the transcriptional machinery presumed to bind to the variable sequence, e.g. a G base in rs10830963 located to an enhancer region binding NEUROD1 in beta cells 
Acknowledgements The views expressed in this article are based on the work of a large group of people who authored references $[2,10]$.

Funding The author has been funded for the work discussed in the article by the Swedish Research Council, The European Foundation for the Study of Diabetes, The Swedish Diabetes and Albert Påhlsson Foundations, Diabetes Wellness and the Medical Faculty in Lund, Sweden.

Duality of interest The author declares that there is no duality of interest associated with this manuscript.

Contribution statement The author was the sole contributor to this paper.

\section{References}

1. Forrestel AC, Miedlich SU, Yurcheshen M, Wittlin SD, Sellix MT (2016) Chronomedicine and type 2 diabetes: shining some light on melatonin. Diabetologia. doi:10.1007/s00125-016-4175-1

2. Tuomi T, Nagorny CL, Singh $P$ et al (2016) Increased melatonin signaling is a risk factor for type 2 diabetes. Cell Metab 23:1067-1077

3. Bonnefond A, Karamitri A, Jockers R, Froguel P (2016) The difficult journey from genome-wide association studies to pathophysiology: the melatonin receptor 1B (MT2) paradigm. Cell Metab 24: 345-347

4. Peschke E, Bahr I, Muhlbauer E (2015) Experimental and clinical aspects of melatonin and clock genes in diabetes. J Pineal Res 59:1-23
5. Muhlbauer E, Peschke E (2007) Evidence for the expression of both the MT1- and in addition, the MT2-melatonin receptor, in the rat pancreas, islet and beta-cell. J Pineal Res 42:105-106

6. Ramracheya RD, Muller DS, Squires PE et al (2008) Function and expression of melatonin receptors on human pancreatic islets. J Pineal Res 44:273-279

7. Blodgett DM, Nowosielska A, Afik S et al (2015) Novel observations from next-generation RNA sequencing of highly purified human adult and fetal islet cell subsets. Diabetes 64:3172-3181

8. Mulder H, Nagorny CLF, Lyssenko V, Groop L (2009) Melatonin receptors in pancreatic islets - good morning to a novel type 2 diabetes gene. Diabetologia 52:1240-1249

9. Peschke E, Bach AG, Muhlbauer E (2006) Parallel signaling pathways of melatonin in the pancreatic beta-cell. J Pineal Res 40:184-191

10. Lyssenko V, Nagorny CL, Erdos MR et al (2009) Common variant in MTNR1B associated with increased risk of type 2 diabetes and impaired early insulin secretion. Nat Genet 41:82-88

11. Bouatia-Naji N, Bonnefond A, Cavalcanti-Proenca C et al (2009) A variant near MTNR1B is associated with increased fasting plasma glucose levels and type 2 diabetes risk. Nat Genet 41:89-94

12. Prokopenko I, Langenberg C, Florez JC et al (2009) Variants in MTNR1B influence fasting glucose levels. Nat Genet 41:77-81

13. Bonnefond A, Clement N, Fawcett K et al (2012) Rare MTNR1B variants impairing melatonin receptor $1 \mathrm{~B}$ function contribute to type 2 diabetes. Nat Genet 44:297-301

14. Gaulton KJ, Ferreira T, Lee Y et al (2015) Genetic fine mapping and genomic annotation defines causal mechanisms at type 2 diabetes susceptibility loci. Nat Genet 47:1415-1425

15. Thomsen SK, McCarthy MI, Gloyn AL (2016) The importance of context: uncovering species- and tissue-specific effects of genetic risk variants for type 2 diabetes. Front Endocrinol 7:112

16. Nagorny CLF, Sathanoori R, Voss U, Mulder H, Wierup N (2011) Distribution of melatonin receptors in murine pancreatic islets. J Pineal Res 50:412-417 